\title{
Packaged Food Supply in Fiji: Nutrient Levels, Compliance with Sodium Targets and Adherence to Labelling Regulations ${ }^{+}$
}

\author{
Maria Shahid 1,*, Gade Waqa 2,*, Arti Pillay ${ }^{3}$, Ateca Kama ${ }^{4}$, Isimeli Tukana 5 , \\ Briar McKenzie ${ }^{1}$, Jacqui Webster ${ }^{1}$ and Claire Johnson ${ }^{1}$
}

1 Food Policy Division, The George Institute for Global Health, University of New South Wales, Sydney, NSW 2042 Australia; bmckenzie@georgeinstitute.org.au (B.M.); jwebster@georgeinstitute.org.au (J.W.); cjohnson@georgeinstitute.org.au (C.J.)

2 Pacific Research Centre for the Prevention of Obesity and Non-Communicable Diseases, Fiji Institute for Pacific Health Research, College of Medicine Nursing and Health Sciences, Fiji National University, Fiji Islands

3 School of Applied Sciences, Fiji National University, Fiji Islands; arti.pillay@fnu.ac.fj

4 National Food and Nutrition Centre, Ministry of Health and Medical Services, Fiji Islands; ateca.kama@yahoo.com

5 Wellness Division, Ministry of Health and Medical Services, Fiji Islands; isimeli.tukana@govnet.gov.fj

* Correspondence: mshahid@georgeinstitute.org.au (M.S.); gade.waqa@fnu.ac.fj (G.W.)

+ Presented at the The 1st International Electronic Conference on Nutrients - Nutritional and Microbiota Effects on Chronic Disease, 2-15 November 2020; Available online: https://iecn2020.sciforum.net/.

Published: 30 October 2020

\begin{abstract}
In response to a high prevalence of NCDs in Fiji and the WHO recommendation to limit the levels of harmful nutrients in packaged foods, the Fijian Government has regulated nutrient labelling and set voluntary sodium reformulation targets. Our aim was to establish a national database of processed and packaged foods and assess compliance to labelling regulations and reformulation targets. Methods: Food labelling and nutrient composition data were collected from the labels of all packaged food products sold at five major supermarket chains in Fiji in 2018. Proportions of products compliant with Fiji labelling regulations, products labelled with sodium and sugar, products compliant with sodium reformulation targets and mean sodium and sugar content were calculated in each food category and for key manufacturers. Results: 5946 packaged food products were surveyed, of which 4278 were included for analysis. Overall compliance with labelling of all required nutrients was low, and only $14 \%$ of packaged foods in 14 major categories met Fiji national labelling regulations. However, sodium was labelled on $95.4 \%$ of products, and sugar labelled on $92.4 \%$. The food group with the highest mean sodium content was convenience foods $(1699 \mathrm{mg} / 100 \mathrm{~g})$ and confectionary $(52.6 \mathrm{~g} / 100 \mathrm{~g})$ had the highest free sugar content. Sixty percent of eligible products met the voluntary Fiji sodium reformulation targets. Conclusions: Improving the nutritional composition of foods and improving labelling has the potential to curb the escalating burden of diet related non-communicable diseases. The food industry has a responsibility to improve the healthiness of its products to enable consumers to make healthy food choices. Our findings indicate ample opportunity for improvements in the labelling and nutritional composition of the Fijian packaged food supply.
\end{abstract}

Keywords: packaged food; sodium; sugar; labelling; Fiji; non-communicable disease

\section{Introduction}


Pacific Island countries are facing a non-communicable disease (NCD) crisis, and unhealthy diets are one of the biggest contributors to the increasing rates of obesity, hypertension and diabetes [1]. NCDs account for about $80 \%$ of all deaths and $50 \%$ of all premature mortality in the Pacific Islands [1]. In Fiji, where more than $30 \%$ of the population are overweight or obese [2], cardiovascular diseases account for one-third of all deaths, and one quarter of deaths are from diabetes. One of the primary reasons for such high rates of NCDs in the country is the transition away from traditional diets, which consisted mainly of fresh fruit, vegetables and fish [3] towards more readily available $[4,5]$ and nutrient poor processed packaged foods that are high in sodium, sugar and fat $[6,7]$.

Pacific Island leaders have been proactive in adopting a range of food policies and regulations to tackle this problem by addressing the increasing consumption of processed foods $[1,8,9]$. This includes taxes on sugar, regulations for sodium levels in processed foods and programs to improve food environments in specific settings such as schools and hospitals $[8,10]$.

The World Health Organization (WHO) recommends limiting the levels of harmful nutrients in products and ensuring that consumers can access and afford healthy food [11]. In Fiji, the National Food and Nutrition Centre (NFNC) was established by the Government in 1982 to coordinate the multi-sectoral efforts needed to address the country's diet-related disease burden. The NFNC has a mission to formulate evidence-based food and nutrition policies through periodic monitoring and evaluation of the food and nutrition environment.

Setting targets for sodium levels in foods is a key component of effective salt (sodium) reduction strategies. In 2010, the Minister for Health and Medical Services in Fiji convened a consultation with key stakeholders from the food industry, government and research groups who agreed on a coordinated strategy to reduce salt intake to $5 \mathrm{gm}$ /day by 2020. The Fiji Salt Action Challenge Strategy was endorsed by way of a Cabinet Submission in 2010, and the NFNC formed a secretariat to provide oversight and momentum.The, NFNC also worked with food industries to understand the food supply in the country, including the sources of available foods and their nutrient content. Such data has the potential to guide interventions to improve diets and enhance health across the population. Based on the contribution of different processed foods to sodium in the diet in the Pacific, the proposed maximum acceptable regional targets for sodium levels in eight selected food categories were developed and agreed by representatives at the Pacific Islands NCD Forum in September 2013 [12]. These were adopted from the Pacific Island targets established the year prior and developed by the Food Taskforce Technical Advisory Group in 2014 [13]. These included: bread, Asian sauces (soy and other Asian sauces), canned fish, canned meat, sausages, snack foods (crisps, extruded snacks, corn chips), biscuits (plain/breakfast, savoury and sweet) and flavoured noodles. The targets were modified to apply specifically to Fiji and finalised through a series of stakeholder and food industry consultations between 2012 and 2014. However, the targets remain voluntary and have not been formally ratified by the Government of Fiji.

All food products sold in Fiji are regulated through the Food Safety Act 2003 [14] and the Food Safety Regulations 2009 [15]. Regulations around the labelling of processed packaged food products require all pre-packaged food produced, processed, packed, distributed, or imported to be labelled in English with the following required nutritional information per $100 \mathrm{~g}$ (or per $100 \mathrm{~mL}$ for liquids) for: energy, protein, fat and carbohydrate. Nutrient declarations are mandatory for foods for which nutrient claims are made and the amount of the nutrient must be expressed as $100 \mathrm{~g}$ or per $100 \mathrm{~mL}$ in metric units and or per serving. In 2014 an amendment of the Food and Safety (Amendment) Regulations 2014 [16] was passed which stipulates mandatory reporting of additional nutrients including trans fatty acids, sodium, sugar, fat, saturated and unsaturated fats to be labelled per $100 \mathrm{~g}$ (or $100 \mathrm{~mL}$ for liquids). Providing this nutrition information on processed packaged foods is important as it allows consumers the ability to assess the nutritional quality of their food. However, to date compliance with these regulations has not been assessed.

The overall aim of this research is to identify opportunities to improve the nutritional quality of the food supply in Fiji. The research comprises a cross-sectional survey of the packaged food supply in Fiji and objectively quantifies the nutritional composition of the main food categories using three criteria; levels of sodium and sugar in packaged food, adherence to national nutrition labelling 
regulations, and compliance of packaged food products with established food reformulation targets. The findings can be used to guide policy decisions related to reformulation activities, front of pack food labelling, import controls, sales taxes and subsidies for healthier products, which in turn will improve the health of the population.

\section{Materials and Methods}

This research encompassed a systematic survey of packaged foods for sale in five major supermarkets (all members of key supermarket chains) in Suva, Fiji. Data were collected between November 2018 and January 2019.

\subsection{Retail Outlets Surveyed}

The stores were purposively selected to ensure that the majority of packaged products in Fiji were included. A total of seven supermarkets were approached and invited, two declined and the remaining five agreed to participate. Supermarkets with more than two branches were selected to ensure more than $70 \%$ of the market was captured. Written permission to collect data was obtained from each store manager prior to commencement of data collection.

\subsection{Packaged Foods Included}

All packaged food products for human consumption that were available for sale in each store during the period of data collection were included. During each product survey, data collection staff captured the barcode and photographed the front of the pack, the nutrition label, manufacturer details, ingredients list and product weight for every packaged food item on every shelf in every outlet surveyed. This was done using a smartphone application developed by The George Institute for Global Health [17] and according to a protocol devised by an international collaborative project designed to document the nutritional composition of packaged foods globally [18]. The images collected by the smartphone application were transmitted to a data management centre in India for processing. Data were uploaded daily into a database which was quality checked, and data was recollected where required.

\subsection{Data Extraction}

The data management centre used a bespoke technology system that enables the systematic, standardised and replicable collection and collation of data describing packaged foods and beverages. Images of food packaging are captured, stored and processed with key data extracted from food labels. The key variables used for the current analysis were the brand name, product name, manufacturer name, serving size and presence of nutritional information per $100 \mathrm{~g}$ (or per $100 \mathrm{~mL}$ for liquids) for energy, protein, carbohydrate, sodium, sugar, total fat, saturated fat and unsaturated fat. Where data was absent from food labels, it was recorded as missing. Different package sizes of the same product were recorded as duplicate items in the database, but each product was included only once in the primary analyses.

\subsection{Categorisation of Foods}

Foods were categorised using the system developed by the Global Food Monitoring Group [18] into 15 major food groups and 58 selected subcategories: (1) bread and bakery products; (2) cereal and grain products; (3) confectionery; (4) convenience foods; (5) dairy and dairy alternatives; (6) edible oils and oil emulsions; (7) eggs; (8) fish and fish products; (9) fruit and vegetables; (10) meat and meat products; (11) non-alcoholic beverages; (12) sauces and spreads; (13) snack foods; (14) sugars, honey and related products; and (15) special foods. Excluded categories were alcoholic beverages, baking powders, chewing gum, cough lollies, eggs, herbs and spices, meal kits, plain teas and coffees, plain waters, sports/protein powders, sugar, sweeteners, vitamins and supplements, yeasts and gelatines since they do not contribute significantly to nutrient intake, nor are manufacturers required to display a Nutritional Information Panel for many of these products. The 
special foods category encompasses baby food and protein and diet bars. This left data for 4278 food products categorised under 14 major categories and 36 subcategories.

\subsection{Manufacturers}

Overall, data for the top 34 manufacturers in Fiji were reported, with manufacturers chosen based on the number of products collected during the survey. Additionally, seven Fiji local manufacturers were identified using in-country expertise. The remaining manufacturers were classified as "other", comprising of smaller manufacturers with a limited number of packaged foods and beverages under their portfolio.

\subsection{Statistical Analysis}

Analyses were conducted across all products and for individual food categories. There were three main sets of analyses: (i) the proportions of packaged foods compliant with Fiji nutrient labelling regulations (i.e., displaying sodium, sugar, energy, protein, carbohydrates, saturated fat, trans fat, monounsaturated fat and polyunsaturated fat), as well as separate proportions for products complying with sodium and sugar labelling; (ii) the average levels of sodium and sugar in packaged foods for which data were available; and (iii) the proportion of packaged foods meeting maximum sodium content targets for the 39 food subcategories for which the targets have been developed. Foods were defined as 'known to meet' the target if the label reported a sodium content that was at or below the specified target.

As a secondary analysis, we compared the proportions of packaged foods compliant with Fiji nutrient labelling regulations across each of the nutrients not examined in the primary analysis (i.e., energy, protein, carbohydrates, saturated fat, trans fat, monounsaturated fat and polyunsaturated fat).

All analyses were done using the statistical software package Stata/IC version 15.1 and figures generated in Microsoft Excel.

\section{Results and Discussion}

\subsection{Nutrition Labelling}

Of the 4278 products analysed, $602(14.1 \%)$ products were fully compliant with Fiji nutrient labelling regulations [Table 1]. Sodium was labelled on $4083(95.4 \%)$ products, and sugar labelled on 3955 (92.4\%). Protein, energy, saturated fat and carbohydrates were also labelled on the majority of products and across most of the food categories, however trans-fat, monounsaturated fat and polyunsaturated fat were only labelled on a minority of products [supplementary Table S1].

Table 1. Proportion of 4278 packaged food products in 2018 meeting Fiji regulation for nutrition labelling and proportion labelled with sodium and sugar, across categories.

\begin{tabular}{lccccccc}
\hline Category & $\begin{array}{c}\text { Total } \\
\text { Products }\end{array}$ & $\begin{array}{c}\text { Meeting Fiji } \\
\text { Nutrition } \\
\text { Labelling } \\
\text { Regulation }\end{array}$ & $\begin{array}{c}\text { Sodium } \\
\text { Labelled }\end{array}$ & $\begin{array}{c}\text { Sugar } \\
\text { Labelled }\end{array}$ \\
\cline { 2 - 8 } & $\boldsymbol{n}$ & $\boldsymbol{n}$ & $\mathbf{\%}$ & $\boldsymbol{n}$ & $\mathbf{\%}$ & $\boldsymbol{n}$ & $\mathbf{\%}$ \\
\hline Bread and bakery products & $\mathbf{5 1 7}$ & $\mathbf{4 5}$ & $\mathbf{8 . 7}$ & $\mathbf{4 9 3}$ & $\mathbf{9 5 . 4}$ & $\mathbf{4 9 6}$ & $\mathbf{9 5 . 9}$ \\
Bread & 54 & 0 & 0.0 & 51 & 94.4 & 54 & 100.0 \\
Cakes, muffins and pastries & 121 & 11 & 9.1 & 121 & 100.0 & 121 & 100.0 \\
Savoury biscuits & 104 & 17 & 16.3 & 103 & 99.0 & 103 & 99.0 \\
Sweet biscuits & 238 & 17 & 7.1 & 218 & 91.6 & 218 & 91.6 \\
Cereal and grain products & 462 & $\mathbf{8 6}$ & $\mathbf{1 8 . 6}$ & $\mathbf{4 4 4}$ & $\mathbf{9 6 . 1}$ & $\mathbf{4 3 4}$ & $\mathbf{9 3 . 9}$ \\
Breakfast cereals & 179 & 53 & 29.6 & 177 & 98.9 & 177 & 98.9 \\
Cereal and nut-based bars & 53 & 6 & 11.3 & 53 & 100.0 & 53 & 100.0
\end{tabular}




\begin{tabular}{|c|c|c|c|c|c|c|c|}
\hline Noodles & 59 & 6 & 10.2 & 55 & 93.2 & 52 & 88.1 \\
\hline Other cereal and grain products & 82 & 11 & 13.4 & 73 & 89.0 & 70 & 85.4 \\
\hline Pasta & 44 & 1 & 2.3 & 42 & 95.5 & 40 & 90.9 \\
\hline Rice & 45 & 9 & 20.0 & 44 & 97.8 & 42 & 93.3 \\
\hline Confectionery & 388 & 10 & 2.6 & 367 & 94.6 & 369 & 95.1 \\
\hline Chocolate and sweets & 364 & 7 & 1.9 & 343 & 94.2 & 345 & 94.8 \\
\hline Jelly & 24 & 3 & 12.5 & 24 & 100.0 & 24 & 100.0 \\
\hline Convenience foods & 60 & 4 & 6.7 & 60 & 100.0 & 60 & 100.0 \\
\hline Ready meals & 15 & 2 & 13.3 & 15 & 100.0 & 15 & 100.0 \\
\hline Soup & 45 & 2 & 4.4 & 45 & 100.0 & 45 & 100.0 \\
\hline Dairy & 348 & 78 & 22.4 & 343 & 98.6 & 341 & 98.0 \\
\hline Cheese & 58 & 4 & 6.9 & 58 & 100.0 & 58 & 100.0 \\
\hline Cream & 26 & 2 & 7.7 & 25 & 96.2 & 26 & 100.0 \\
\hline Desserts & 11 & 1 & 9.1 & 11 & 100.0 & 11 & 100.0 \\
\hline Ice cream and edible ices & 112 & 17 & 15.2 & 110 & 98.2 & 110 & 98.2 \\
\hline Milk & 87 & 33 & 37.9 & 85 & 97.7 & 82 & 94.3 \\
\hline Yoghurt and yoghurt drinks & 54 & 21 & 38.9 & 54 & 100.0 & 54 & 100.0 \\
\hline Edible oils and oil emulsions & 105 & 58 & 55.2 & 99 & 94.3 & 88 & 83.8 \\
\hline Fish and fish products & 116 & 41 & 35.3 & 114 & 98.3 & 104 & 89.7 \\
\hline Fruit and vegetables & 678 & 77 & 11.4 & 659 & 97.2 & 644 & 95.0 \\
\hline Fruit & 125 & 6 & 4.8 & 123 & 98.4 & 122 & 97.6 \\
\hline Jam and marmalades & 81 & 13 & 16.0 & 77 & 95.1 & 75 & 92.6 \\
\hline Nuts and seeds & 140 & 27 & 19.3 & 138 & 98.6 & 132 & 94.3 \\
\hline Vegetables & 332 & 31 & 9.3 & 321 & 96.7 & 315 & 94.9 \\
\hline Meat and meat products & 105 & 5 & 4.8 & 99 & 94.3 & 70 & 66.7 \\
\hline Non-alcoholic beverages & 487 & 38 & 7.8 & 434 & 89.1 & 451 & 92.6 \\
\hline Beverage mixes & 38 & 0 & 0.0 & 37 & 97.4 & 35 & 92.1 \\
\hline Coffee and tea & 51 & 4 & 7.8 & 46 & 90.2 & 44 & 86.3 \\
\hline Cordials & 26 & 4 & 15.4 & 14 & 53.8 & 22 & 84.6 \\
\hline Electrolyte and energy drinks & 32 & 0 & 0.0 & 27 & 84.4 & 27 & 84.4 \\
\hline Fruit and vegetable juices & 228 & 14 & 6.1 & 202 & 88.6 & 215 & 94.3 \\
\hline Soft drinks & 94 & 12 & 12.8 & 92 & 97.9 & 92 & 97.9 \\
\hline Waters (flavoured) & 18 & 4 & 22.2 & 16 & 88.9 & 16 & 88.9 \\
\hline Sauces, dressings, spreads and dips & 628 & 42 & 6.7 & 610 & 97.1 & 574 & 91.4 \\
\hline Mayonnaise and salad dressings & 57 & 6 & 10.5 & 57 & 100.0 & 53 & 93.0 \\
\hline Sauces & 416 & 15 & 3.6 & 401 & 96.4 & 370 & 88.9 \\
\hline Spreads and dips & 155 & 21 & 13.5 & 152 & 98.1 & 151 & 97.4 \\
\hline Snackfoods & 290 & 106 & 36.6 & 268 & 92.4 & 240 & 82.8 \\
\hline Special foods & 26 & 0 & 0.0 & 25 & 96.2 & 20 & 76.9 \\
\hline Sugars, honey and related products & 68 & 15 & 22.1 & 68 & 100.0 & 64 & 94.1 \\
\hline Dessert additions and toppings & 42 & 9 & 21.4 & 42 & 100.0 & 41 & 97.6 \\
\hline Honey and syrup & 26 & 6 & 23.1 & 26 & 100.0 & 23 & 88.5 \\
\hline Total & 4278 & 605 & 14.1 & 4083 & 95.4 & 3955 & 92.4 \\
\hline
\end{tabular}

Of the 34 top manufacturers selected for analyses, 21 complied with sodium labelling across their products, and 19 complied with sugar labelling [Table 2]. The manufacturer with the lowest compliance to both sodium and sugar labelling was Carpenters Fiji PTE Limited (79.5\% and 74.4\% respectively). PepsiCo had the highest proportion of products meeting Fiji nutrient labelling regulations (33 $(64.7 \%))$. 
Table 2. Proportion of 4278 packaged food products in 2018 meeting Fiji regulation for nutrition labelling and proportion labelled with sodium and sugar, across manufacturers.

\begin{tabular}{|c|c|c|c|c|c|c|c|}
\hline \multirow[t]{2}{*}{ Manufacturer } & \multirow{2}{*}{$\begin{array}{c}\begin{array}{c}\text { Total } \\
\text { Products }\end{array} \\
n \\
\end{array}$} & \multicolumn{2}{|c|}{$\begin{array}{l}\text { Meeting Fiji Nutrition } \\
\text { Labelling Regulation }\end{array}$} & \multicolumn{2}{|c|}{$\begin{array}{l}\text { Sodium } \\
\text { Labelled }\end{array}$} & \multicolumn{2}{|c|}{$\begin{array}{c}\text { Sugar } \\
\text { Labelled }\end{array}$} \\
\hline & & $n$ & $\%$ & $n$ & $\%$ & $n$ & $\%$ \\
\hline Ashabhai \& Co. & 68 & 41 & 60.3 & 67 & 98.5 & 66 & 97.1 \\
\hline CJ Patel Group & 23 & 9 & 39.1 & 23 & 100.0 & 22 & 95.7 \\
\hline Campbell Arnott's & 45 & 4 & 8.9 & 43 & 95.6 & 43 & 95.6 \\
\hline Carpenters Fiji PTE & 39 & 8 & 20.5 & 31 & 79.5 & 29 & 74.4 \\
\hline Coca Cola Amatil & 40 & 0 & 0.0 & 40 & 100.0 & 40 & 100.0 \\
\hline Desai Brothers & 37 & 0 & 0.0 & 34 & 91.9 & 37 & 100.0 \\
\hline Eco Farms & 30 & 1 & 3.3 & 30 & 100.0 & 30 & 100.0 \\
\hline FMF Foods & 51 & 24 & 47.1 & 50 & 98.0 & 50 & 98.0 \\
\hline Food Processors (Fiji) & 10 & 0 & 0.0 & 10 & 100.0 & 8 & 80.0 \\
\hline Foods Pacific Group & 16 & 2 & 12.5 & 16 & 100.0 & 16 & 100.0 \\
\hline General Mills & 54 & 1 & 1.9 & 54 & 100.0 & 54 & 100.0 \\
\hline George Weston Foods & 64 & 2 & 3.1 & 64 & 100.0 & 63 & 98.4 \\
\hline Goodman Fielder & 111 & 36 & 32.4 & 110 & 99.1 & 89 & 80.2 \\
\hline Heinz & 89 & 10 & 11.2 & 88 & 98.9 & 89 & 100.0 \\
\hline IGA & 52 & 3 & 5.8 & 52 & 100.0 & 52 & 100.0 \\
\hline Kellogg's & 36 & 4 & 11.1 & 36 & 100.0 & 36 & 100.0 \\
\hline Lion Dairy \& Drinks & 33 & 0 & 0.0 & 33 & 100.0 & 33 & 100.0 \\
\hline Lolliland & 34 & 0 & 0.0 & 32 & 94.1 & 34 & 100.0 \\
\hline Mars & 82 & 0 & 0.0 & 78 & 95.1 & 78 & 95.1 \\
\hline Mondelez & 92 & 14 & 15.2 & 92 & 100.0 & 91 & 98.9 \\
\hline Motibhai Group & 38 & 0 & 0.0 & 38 & 100.0 & 37 & 97.4 \\
\hline Nestle & 82 & 15 & 18.3 & 78 & 95.1 & 75 & 91.5 \\
\hline Oriental Merchant & 40 & 2 & 5.0 & 39 & 97.5 & 39 & 97.5 \\
\hline Parmalat & 41 & 1 & 2.4 & 41 & 100.0 & 41 & 100.0 \\
\hline PepsiCo & 51 & 33 & 64.7 & 51 & 100.0 & 51 & 100.0 \\
\hline Punjas & 100 & 37 & 37.0 & 97 & 97.0 & 99 & 99.0 \\
\hline SPC Ardmona & 35 & 0 & 0.0 & 35 & 100.0 & 35 & 100.0 \\
\hline San Remo & 33 & 0 & 0.0 & 33 & 100.0 & 33 & 100.0 \\
\hline Sanitarium & 36 & 19 & 52.8 & 36 & 100.0 & 36 & 100.0 \\
\hline Simplot & 74 & 28 & 37.8 & 74 & 100.0 & 74 & 100.0 \\
\hline SunRice & 41 & 5 & 12.2 & 41 & 100.0 & 39 & 95.1 \\
\hline Unilever & 40 & 11 & 27.5 & 40 & 100.0 & 40 & 100.0 \\
\hline Whittaker's & 31 & 0 & 0.0 & 31 & 100.0 & 31 & 100.0 \\
\hline Woolworths & 143 & 15 & 10.5 & 142 & 99.3 & 143 & 100.0 \\
\hline All other manufacturers & 2487 & 280 & 11.3 & 2324 & 93.4 & 2222 & 89.3 \\
\hline Total & 4278 & 605 & 14.1 & 4083 & 95.4 & 3955 & 92.4 \\
\hline
\end{tabular}

\subsection{Sodium and Sugar Content}

There was significant variability across all categories and subcategories for both sodium and sugar content [Table 3]. Food categories with the highest mean sodium content were 'convenience foods' (1699 mg/100 g) and 'sauces, dressings, spreads and dips' (1422 mg/100 g). Unsurprisingly, categories with the lowest mean sodium content were 'sugars, honey and related products' (36 $\mathrm{mg} / 100 \mathrm{~g}$ ) and 'non-alcoholic beverages' (49 mg/100 g). There was significant variability across all categories and subcategories. 
Table 3. Mean sodium and sugar content of food products, surveyed in Fiji in 2018.

\begin{tabular}{|c|c|c|c|c|c|c|c|c|}
\hline \multirow[b]{2}{*}{ Category } & \multicolumn{4}{|c|}{ Sodium $(\mathrm{mg} / 100 \mathrm{~g})$} & \multicolumn{4}{|c|}{ Sugar $(g / 100 g)$} \\
\hline & $n$ & $\begin{array}{c}\text { mean } \\
(S D)\end{array}$ & $\begin{array}{c}\text { median } \\
(I Q R)\end{array}$ & range & $n$ & $\begin{array}{c}\text { mean } \\
(S D)\end{array}$ & $\begin{array}{c}\text { median } \\
(I Q R)\end{array}$ & range \\
\hline Bread and bakery products & 493 & $\begin{array}{c}415 \\
(301)\end{array}$ & $\begin{array}{c}335 \\
(306)\end{array}$ & 0-2000 & 496 & $\begin{array}{c}24.0 \\
(16.6)\end{array}$ & $\begin{array}{c}26.7 \\
(32.3)\end{array}$ & $0.0-64.9$ \\
\hline Bread & 51 & $\begin{array}{c}611 \\
(456)\end{array}$ & $\begin{array}{c}486 \\
(415)\end{array}$ & $2-2000$ & 54 & $5.1(8.3)$ & $\begin{array}{c}2.5 \\
(4.6)\end{array}$ & $0.0-42.8$ \\
\hline Cakes, muffins and pastries & 121 & $\begin{array}{c}450 \\
(246)\end{array}$ & $\begin{array}{c}394 \\
(393)\end{array}$ & 0-1184 & 121 & $\begin{array}{c}33.7 \\
(15.7)\end{array}$ & $\begin{array}{c}40.2 \\
(20.4)\end{array}$ & $0.0-64.9$ \\
\hline Savoury biscuits & 103 & $\begin{array}{c}631 \\
(310)\end{array}$ & $\begin{array}{c}556 \\
(370)\end{array}$ & $1-1600$ & 103 & $5.8(8.2)$ & $\begin{array}{c}3.4 \\
(4.9)\end{array}$ & $0.0-51.4$ \\
\hline Sweet biscuits & 218 & $\begin{array}{c}248 \\
(140)\end{array}$ & $\begin{array}{c}239 \\
(182)\end{array}$ & $17-837$ & 218 & $\begin{array}{l}31.9 \\
(9.3)\end{array}$ & $\begin{array}{c}32.9 \\
(12.2)\end{array}$ & $0.0-50.0$ \\
\hline Cereal and grain products & 444 & $\begin{array}{c}347 \\
(872)\end{array}$ & $61(361)$ & $0-14,500$ & 434 & $\begin{array}{c}11.0 \\
(11.8)\end{array}$ & $\begin{array}{c}6.0 \\
(17.4)\end{array}$ & $0.0-68.2$ \\
\hline Breakfast cereals & 177 & $\begin{array}{c}225 \\
(214)\end{array}$ & $\begin{array}{c}170 \\
(341)\end{array}$ & 0-1000 & 177 & $\begin{array}{c}16.7 \\
(10.2)\end{array}$ & $\begin{array}{c}16.7 \\
(14.5)\end{array}$ & $0.0-41.3$ \\
\hline Cereal and nut-based bars & 53 & $\begin{array}{c}106 \\
(121)\end{array}$ & $57(137)$ & $4-467$ & 53 & $\begin{array}{c}25.0 \\
(13.1)\end{array}$ & $\begin{array}{c}21.6 \\
(10.5)\end{array}$ & $0.0-68.2$ \\
\hline Noodles & 55 & $\begin{array}{l}1179 \\
(846)\end{array}$ & $\begin{array}{c}1340 \\
(1604)\end{array}$ & $0-2880$ & 52 & $3.9(4.1)$ & $\begin{array}{c}3.1 \\
(4.8)\end{array}$ & $0.0-24.6$ \\
\hline $\begin{array}{l}\text { Other cereal and grain } \\
\text { products }\end{array}$ & 73 & $\begin{array}{c}528 \\
(1801)\end{array}$ & $17(551)$ & $0-14,500$ & 70 & $2.6(3.9)$ & $\begin{array}{l}1.8 \\
(2.5)\end{array}$ & $0.0-25.0$ \\
\hline Pasta & 42 & $\begin{array}{c}87 \\
(180)\end{array}$ & $29(27)$ & $0-957$ & 40 & $2.5(1.7)$ & $\begin{array}{c}2.5 \\
(2.0)\end{array}$ & $0.0-7.1$ \\
\hline Rice & 44 & $\begin{array}{c}33 \\
(133)\end{array}$ & $2(4)$ & $0-691$ & 42 & $0.4(0.5)$ & $\begin{array}{c}0.1 \\
(1.0)\end{array}$ & $0.0-1.8$ \\
\hline Confectionery & 367 & $\begin{array}{c}112 \\
(229)\end{array}$ & $62(89)$ & $0-3000$ & 369 & $\begin{array}{c}52.6 \\
(20.2)\end{array}$ & $\begin{array}{c}52.6 \\
(20.2)\end{array}$ & $\begin{array}{c}0.0- \\
100.0\end{array}$ \\
\hline Chocolate and sweets & 343 & $\begin{array}{c}115 \\
(235)\end{array}$ & $63(88)$ & $0-3000$ & 345 & $\begin{array}{c}52.1 \\
(19.7)\end{array}$ & $\begin{array}{c}52.1 \\
(19.0)\end{array}$ & $\begin{array}{c}0.0- \\
100.0\end{array}$ \\
\hline Jelly & 24 & $\begin{array}{c}66 \\
(76)\end{array}$ & $33(101)$ & $0-311$ & 24 & $\begin{array}{c}58.7 \\
(25.3)\end{array}$ & $\begin{array}{c}62.5 \\
(41.0)\end{array}$ & $1.0-89.0$ \\
\hline Convenience foods & 60 & $\begin{array}{c}1699 \\
(3765)\end{array}$ & $\begin{array}{c}300 \\
(1098)\end{array}$ & $0-26000$ & 60 & $4.3(4.9)$ & $\begin{array}{c}2.9 \\
(4.6)\end{array}$ & $0.2-25.0$ \\
\hline Ready meals & 15 & $\begin{array}{c}621 \\
(658)\end{array}$ & $\begin{array}{c}600 \\
(932)\end{array}$ & $0-2432$ & 15 & $3.0(2.1)$ & $\begin{array}{c}2.5 \\
(3.0)\end{array}$ & $0.5-7.8$ \\
\hline Soup & 45 & $\begin{array}{c}2058 \\
(4283)\end{array}$ & $\begin{array}{c}298 \\
(1585)\end{array}$ & $0-26000$ & 45 & $4.8(5.5)$ & $\begin{array}{c}3.0 \\
(6.4)\end{array}$ & $0.2-25.0$ \\
\hline Dairy & 343 & $\begin{array}{c}202 \\
(352)\end{array}$ & $52(87)$ & 0-1903 & 341 & $\begin{array}{c}14.7 \\
(16.0)\end{array}$ & $\begin{array}{c}10.4 \\
(19.6)\end{array}$ & $0.0-83.5$ \\
\hline Cheese & 58 & $\begin{array}{c}853 \\
(432)\end{array}$ & $\begin{array}{c}712 \\
(561)\end{array}$ & 125-1903 & 58 & $2.8(6.6)$ & $\begin{array}{c}1.0 \\
(1.2)\end{array}$ & $0.0-45.0$ \\
\hline Cream & 25 & $\begin{array}{c}154 \\
(133)\end{array}$ & $\begin{array}{c}191 \\
(164)\end{array}$ & $0-470$ & 26 & $\begin{array}{c}35.5 \\
(30.1)\end{array}$ & $\begin{array}{c}53.0 \\
(57.2)\end{array}$ & $1.0-83.5$ \\
\hline Desserts & 11 & $\begin{array}{c}198 \\
(234)\end{array}$ & $\begin{array}{c}135 \\
(174)\end{array}$ & $22-700$ & 11 & $\begin{array}{c}11.4 \\
(12.5)\end{array}$ & $\begin{array}{c}4.4 \\
(27.1)\end{array}$ & $0.0-27.9$ \\
\hline Ice cream and edible ices & 110 & $\begin{array}{c}41 \\
(40)\end{array}$ & $37(40)$ & $0-261$ & 110 & $\begin{array}{c}21.7 \\
(10.6)\end{array}$ & $\begin{array}{c}21.8 \\
(11.2)\end{array}$ & $2.1-67.0$ \\
\hline Milk & 85 & $\begin{array}{c}70 \\
(72)\end{array}$ & $47(23)$ & $8-390$ & 82 & $\begin{array}{c}10.2 \\
(14.3)\end{array}$ & $\begin{array}{c}5.0 \\
(6.6)\end{array}$ & $0.0-56.3$ \\
\hline $\begin{array}{l}\text { Yoghurt and yoghurt } \\
\text { drinks }\end{array}$ & 54 & $\begin{array}{c}58 \\
(18)\end{array}$ & $52(10)$ & $22-118$ & 54 & $\begin{array}{l}10.5 \\
(8.4)\end{array}$ & $\begin{array}{c}10.5 \\
(16.7)\end{array}$ & $0.0-31.3$ \\
\hline $\begin{array}{l}\text { Edible oils and oil } \\
\text { emulsions }\end{array}$ & 99 & $\begin{array}{c}195 \\
(346)\end{array}$ & $0(360)$ & $0-1200$ & 88 & $0.3(0.5)$ & $\begin{array}{c}0.0 \\
(0.6)\end{array}$ & $0.0-3.0$ \\
\hline Fish and fish products & 114 & $\begin{array}{c}602 \\
(951)\end{array}$ & $\begin{array}{c}400 \\
(168)\end{array}$ & $10-7533$ & 104 & $2.0(5.2)$ & $\begin{array}{l}1.0 \\
(2.0)\end{array}$ & $0.0-48.0$ \\
\hline
\end{tabular}




\begin{tabular}{|c|c|c|c|c|c|c|c|c|}
\hline Fruit and vegetables & 659 & $\begin{array}{c}317 \\
(694)\end{array}$ & $32(304)$ & $0-5333$ & 644 & $\begin{array}{c}16.4 \\
(23.1)\end{array}$ & $\begin{array}{c}4.5 \\
(14.8)\end{array}$ & $0.0-75.0$ \\
\hline Fruit & 123 & $\begin{array}{c}37 \\
(195)\end{array}$ & $6(14)$ & $0-2100$ & 122 & $\begin{array}{c}30.5 \\
(24.5)\end{array}$ & $\begin{array}{c}17.0 \\
(43.3)\end{array}$ & $0.0-75.0$ \\
\hline Jam and marmalades & 77 & $\begin{array}{c}13 \\
(18)\end{array}$ & $9(10)$ & $0-130$ & 75 & $\begin{array}{l}62.4 \\
(4.6)\end{array}$ & $\begin{array}{l}63.4 \\
(4.4)\end{array}$ & $\begin{array}{l}48.6- \\
70.0\end{array}$ \\
\hline Nuts and seeds & 138 & $\begin{array}{c}190 \\
(223)\end{array}$ & $65(316)$ & 0-1037 & 132 & $\begin{array}{c}8.6 \\
(11.7)\end{array}$ & $\begin{array}{c}4.7 \\
(3.6)\end{array}$ & $0.0-55.0$ \\
\hline Vegetables & 321 & $\begin{array}{c}552 \\
(917)\end{array}$ & $\begin{array}{c}240 \\
(589)\end{array}$ & $0-5333$ & 315 & $3.3(4.9)$ & $\begin{array}{c}2.0 \\
(3.3)\end{array}$ & $0.0-49.7$ \\
\hline Meat and meat products & 99 & $\begin{array}{c}679 \\
(508)\end{array}$ & $\begin{array}{c}632 \\
(546)\end{array}$ & 30-1960 & 70 & $0.7(1.1)$ & $\begin{array}{c}0.3 \\
(0.9)\end{array}$ & $0.0-7.6$ \\
\hline Non-alcoholic beverages & 434 & $\begin{array}{c}49 \\
(147)\end{array}$ & $8(18)$ & $0-1300$ & 451 & $\begin{array}{l}14.8 \\
(20.1)\end{array}$ & $\begin{array}{l}10.3 \\
(4.8)\end{array}$ & $0.0-98.7$ \\
\hline Beverage mixes & 37 & $\begin{array}{c}255 \\
(353)\end{array}$ & $30(388)$ & $0-1300$ & 35 & $\begin{array}{c}39.6 \\
(40.1)\end{array}$ & $\begin{array}{c}12.0 \\
(77.2)\end{array}$ & $0.1-93.3$ \\
\hline Coffee and tea & 46 & $\begin{array}{c}113 \\
(208)\end{array}$ & $39(68)$ & $0-950$ & 44 & $\begin{array}{c}27.7 \\
(33.2)\end{array}$ & $\begin{array}{c}7.1 \\
(54.9)\end{array}$ & $0.0-98.7$ \\
\hline Cordials & 14 & $\begin{array}{c}23 \\
(35)\end{array}$ & $6(40)$ & $1-130$ & 22 & $\begin{array}{c}35.0 \\
(31.0)\end{array}$ & $\begin{array}{c}38.8 \\
(53.4)\end{array}$ & $0.0-79.8$ \\
\hline $\begin{array}{l}\text { Electrolyte and energy } \\
\text { drinks }\end{array}$ & 27 & $\begin{array}{c}46 \\
(28)\end{array}$ & $46(23)$ & $0-110$ & 27 & $6.8(4.1)$ & $\begin{array}{c}6.0 \\
(4.8)\end{array}$ & $0.0-13.9$ \\
\hline Fruit and vegetable juices & 202 & $\begin{array}{c}21 \\
(63)\end{array}$ & $6(10)$ & $0-500$ & 215 & $\begin{array}{l}10.5 \\
(6.4)\end{array}$ & $\begin{array}{l}10.5 \\
(2.5)\end{array}$ & $0.0-94.1$ \\
\hline Soft drinks & 92 & $8(5)$ & $7(6)$ & $0-23$ & 92 & $8.8(4.3)$ & $\begin{array}{l}10.5 \\
(3.5)\end{array}$ & $0.0-14.0$ \\
\hline Waters (flavoured) & 16 & $\begin{array}{c}13 \\
(28)\end{array}$ & $1(18)$ & $0-113$ & 16 & $4.7(3.5)$ & $\begin{array}{c}5.2 \\
(5.0)\end{array}$ & $0.0-12.8$ \\
\hline $\begin{array}{l}\text { Sauces, dressings, spreads } \\
\text { and dips }\end{array}$ & 610 & $\begin{array}{c}1422 \\
(1982)\end{array}$ & $\begin{array}{c}670 \\
(1046)\end{array}$ & $0-16667$ & 574 & $\begin{array}{c}14.2 \\
(15.6)\end{array}$ & $\begin{array}{c}8.1 \\
(17.3)\end{array}$ & $0.0-79.0$ \\
\hline $\begin{array}{l}\text { Mayonnaise and salad } \\
\text { dressings }\end{array}$ & 57 & $\begin{array}{c}713 \\
(625)\end{array}$ & $\begin{array}{c}756 \\
(763)\end{array}$ & $0-3400$ & 53 & $\begin{array}{c}12.1 \\
(10.5)\end{array}$ & $\begin{array}{c}13.0 \\
(13.0)\end{array}$ & $0.0-50.0$ \\
\hline Sauces & 401 & $\begin{array}{l}1710 \\
(2245)\end{array}$ & $\begin{array}{c}833 \\
(1616)\end{array}$ & $0-16667$ & 370 & $\begin{array}{c}13.5 \\
(14.1)\end{array}$ & $\begin{array}{c}7.1 \\
(18.1)\end{array}$ & $0.0-79.0$ \\
\hline Spreads and dips & 152 & $\begin{array}{c}929 \\
(1299)\end{array}$ & $\begin{array}{c}437 \\
(632)\end{array}$ & $0-7100$ & 151 & $\begin{array}{c}16.6 \\
(19.8)\end{array}$ & $\begin{array}{c}9.2 \\
(18.1)\end{array}$ & $0.0-77.3$ \\
\hline Snackfoods & 268 & $\begin{array}{c}690 \\
(381)\end{array}$ & $\begin{array}{l}670 \\
(351)\end{array}$ & $1-4000$ & 240 & $4.9(5.8)$ & $\begin{array}{c}3.6 \\
(3.6)\end{array}$ & $0.0-45.0$ \\
\hline Special foods & 25 & $\begin{array}{c}103 \\
(146)\end{array}$ & $21(168)$ & $1-533$ & 20 & $6.3(7.7)$ & $\begin{array}{c}5.3 \\
(6.1)\end{array}$ & $0.0-35.0$ \\
\hline $\begin{array}{l}\text { Sugars, honey and related } \\
\text { products }\end{array}$ & 68 & $\begin{array}{c}41 \\
(66)\end{array}$ & $11(46)$ & $0-300$ & 64 & $\begin{array}{c}44.2 \\
(26.1)\end{array}$ & $\begin{array}{c}50.0 \\
(46.3)\end{array}$ & $1.0-83.3$ \\
\hline $\begin{array}{l}\text { Dessert additions and } \\
\text { toppings }\end{array}$ & 42 & $\begin{array}{c}43 \\
(69)\end{array}$ & $10(64)$ & $0-300$ & 41 & $\begin{array}{c}46.4 \\
(25.4)\end{array}$ & $\begin{array}{c}50.0 \\
(24.6)\end{array}$ & $1.0-83.3$ \\
\hline Honey and syrup & 26 & $\begin{array}{c}39 \\
(62)\end{array}$ & $13(25)$ & $0-207$ & 23 & $\begin{array}{c}40.2 \\
(27.4)\end{array}$ & $\begin{array}{c}28.0 \\
(50.2)\end{array}$ & 7.5-80.9 \\
\hline Total & 4083 & $\begin{array}{c}493 \\
(1110)\end{array}$ & $\begin{array}{c}183 \\
(516)\end{array}$ & $0-26,000$ & $\begin{array}{c}3,95 \\
5\end{array}$ & $\begin{array}{c}18.0 \\
(21.5)\end{array}$ & $\begin{array}{c}8.7 \\
(24.9)\end{array}$ & $\begin{array}{c}0.0- \\
100.0\end{array}$ \\
\hline
\end{tabular}

Food categories with the highest mean sugar content were 'confectionery' (52.6 g/100 g) and 'sugars, honey and related products' $(44.2 \mathrm{~g} / 100 \mathrm{~g})$. Categories with the lowest mean sugar content were 'edible oils and oil emulsions' $(0.3 \mathrm{~g} / 100 \mathrm{~g})$ and 'meat and meat products' ( $0.7 \mathrm{~g} / 100 \mathrm{~g})$.

\subsection{Assessing against Reformulation Targets}

Of the 1188 products with sodium reformulation targets, 707 (59.5\%) met the relevant target. The highest compliance was observed for 'bacon' (100\%) and 'sweet, filled biscuits' (90.4\%), which had reformulation targets of $1210 \mathrm{mg} / 100 \mathrm{~g}$ and $450 \mathrm{mg} / 100 \mathrm{~g}$ respectively [Table 4]. The categories of 
'canned meat', 'meat-free products', 'shrimps/prawns peeled cooked/crumbed' and 'squid/calamari' did not have any products meeting sodium reformulation targets.

Table 4. Compliance of targeted food categories with voluntary Fijian sodium reformulation targets.

\begin{tabular}{|c|c|c|c|c|c|}
\hline \multirow[t]{2}{*}{ Category } & \multirow[t]{2}{*}{ Sub Category } & \multirow{2}{*}{$\begin{array}{c}\text { Reformulation } \\
\text { Target } \\
\text { (mg/100 g) }\end{array}$} & \multirow{2}{*}{$\begin{array}{c}\begin{array}{c}\text { Total } \\
\text { Products }\end{array} \\
n\end{array}$} & \multicolumn{2}{|c|}{$\begin{array}{c}\text { Meeting } \\
\text { Target }\end{array}$} \\
\hline & & & & $n$ & $\%$ \\
\hline \multirow{4}{*}{ Biscuits } & Plain, dry & 610 & 40 & 28 & 70.0 \\
\hline & Savoury & 800 & 63 & 41 & 65.1 \\
\hline & Sweet, filled & 450 & 135 & 122 & 90.4 \\
\hline & Sweet, unfilled & 450 & 100 & 81 & 81.0 \\
\hline \multirow{7}{*}{ Canned fish } & Fish finger/fillet & 350 & 7 & 3 & 42.9 \\
\hline & Mackerel & 420 & 29 & 19 & 65.5 \\
\hline & Salmon-pink & 430 & 11 & 9 & 81.8 \\
\hline & Sardines & 360 & 11 & 7 & 63.6 \\
\hline & $\begin{array}{l}\text { Shrimps/prawns } \\
\text { cooked/crumbed }\end{array}$ & 350 & 2 & 0 & 0.0 \\
\hline & Squid/calamari & 350 & 1 & 0 & 0.0 \\
\hline & Tuna & 390 & 34 & 14 & 41.2 \\
\hline \multirow{8}{*}{$\begin{array}{l}\text { Meat \& other } \\
\text { products }\end{array}$} & Bacon & 1210 & 1 & 1 & 100.0 \\
\hline & Canned meat & 540 & 11 & 0 & 0.0 \\
\hline & Luncheon meat & 1030 & 6 & 3 & 50.0 \\
\hline & Meat-free products & 480 & 1 & 0 & 0.0 \\
\hline & Salami & 1400 & 6 & 1 & 16.7 \\
\hline & Sausages - pre-cooked & 650 & 8 & 1 & 12.5 \\
\hline & Sausages - uncooked & 650 & 23 & 8 & 34.8 \\
\hline & Sliced meat (ham, beef, chicken) & 650 & 8 & 1 & 12.5 \\
\hline Noodles & Instant flavoured assorted - dry & 370 & 40 & 8 & 20.0 \\
\hline \multirow{12}{*}{$\begin{array}{l}\text { Sauces } \\
\text { spreads }\end{array}$} & Asian sauces & 4840 & 92 & 53 & 57.6 \\
\hline & Chilli sauce & 1600 & 43 & 22 & 51.2 \\
\hline & Gravy Stock & 540 & 33 & 24 & 72.7 \\
\hline & Marinade & 1600 & 16 & 6 & 37.5 \\
\hline & Mayonnaise & 650 & 16 & 7 & 43.8 \\
\hline & Meal-based - curry paste & 490 & 9 & 3 & 33.3 \\
\hline & Meal-based sauces-other & 800 & 70 & 43 & 61.4 \\
\hline & Meat accompaniment & 600 & 15 & 13 & 86.7 \\
\hline & Mustard & 1910 & 25 & 20 & 80.0 \\
\hline & Pasta sauce & 450 & 40 & 30 & 75.0 \\
\hline & Salad dressing & 940 & 28 & 18 & 64.3 \\
\hline & Tomato sauce & 750 & 38 & 13 & 34.2 \\
\hline \multirow{7}{*}{ Snacks } & Corn chips & 560 & 17 & 9 & 52.9 \\
\hline & Extruded & 750 & 78 & 35 & 44.9 \\
\hline & Other: dalo, cassava chips etc & 560 & 8 & 7 & 87.5 \\
\hline & Other: rice crackers, popcorn etc & 650 & 15 & 2 & 13.3 \\
\hline & Potato crisps & 600 & 65 & 36 & 55.4 \\
\hline & Salt \& vinegar & 1000 & 8 & 5 & 62.5 \\
\hline & Snack packs-bhujas etc & 650 & 35 & 14 & 40.0 \\
\hline Total & & & 1188 & 707 & 59.5 \\
\hline
\end{tabular}

Amongst manufacturers with at least three products eligible for voluntary sodium reformulation targets, Food Processors (Fiji) had the highest proportion of products meeting the 
targets $(\mathrm{n}(\%)=7(100 \%))$ (Figure 1). None of the six Desai Brothers products, which were all in the sauces and spreads category, met the salt targets.

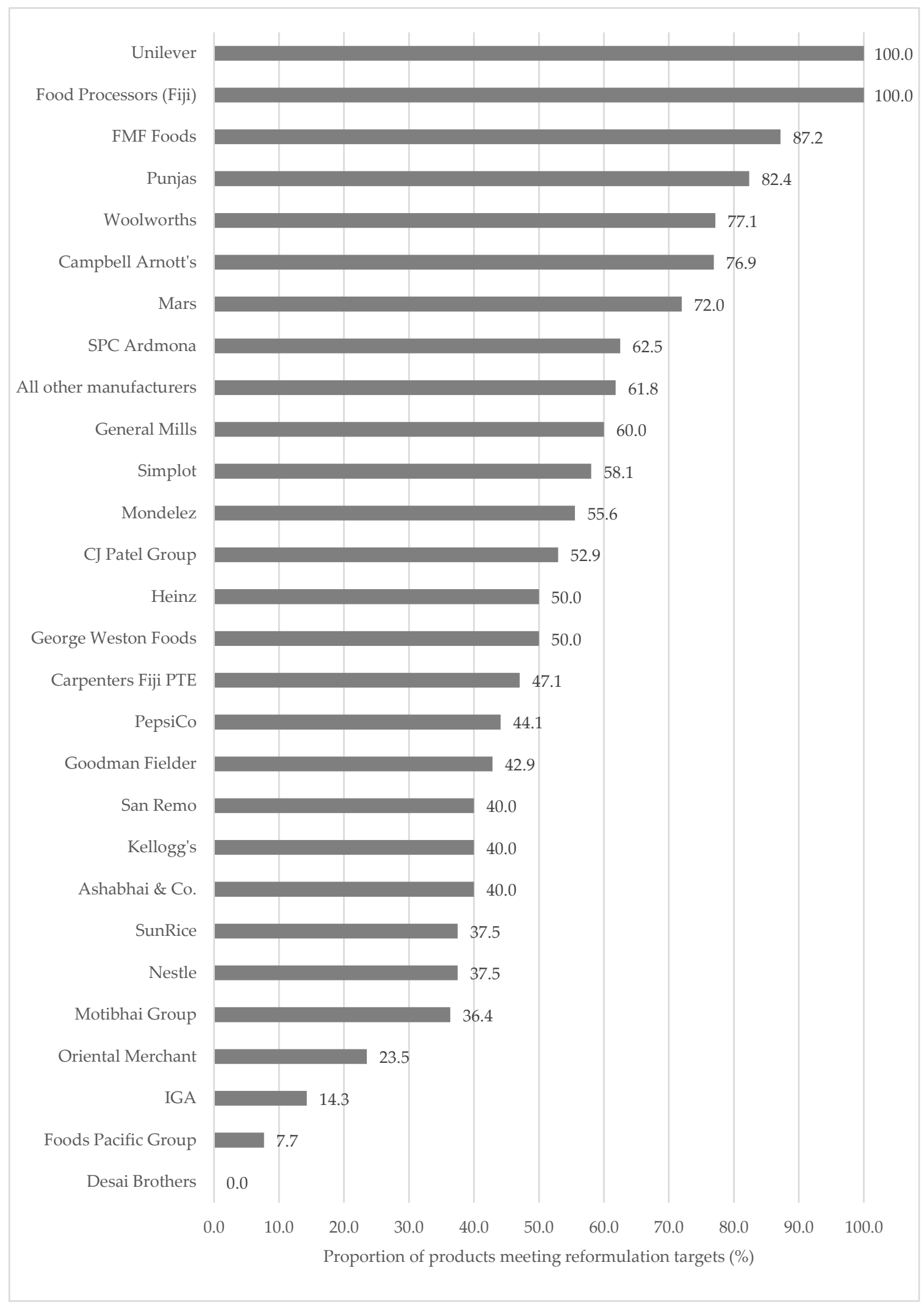

Figure 1. Compliance of International and National manufacturers producing eligible products with voluntary Fijian sodium reformulation targets. 


\section{Conclusions}

The packaged foods and beverages available in Fiji include a high proportion of products that contain high levels of sodium and sugar, and whilst the proportion of products labelling these nutrients is high, overall compliance to labelling regulations is low. The Fijian reformulation targets are currently unratified, and progress towards meeting the targets varies between food categories. The government of Fiji needs to formally adopt the targets and develop targets for sugar based on WHO recommendations. Additionally, ongoing, sustainable political support for sodium and sugar reduction strategies is urgently required. The food industry has a responsibility to consumers to improve the healthiness of its products and make it easier to identify healthier options. There are multiple ways to achieve this, but rapid and substantive gains will be greatly facilitated with government leadership. Actions that improve the quality of the food supply have the potential to reduce overweight, obesity and premature death and disability for hundreds of thousands of Fijians.

Funding: This work was supported by a DFAT Food Innovation Grant. The funders had no role in the design, analysis or writing of this article. This project is supported by an NHMRC Project Grant (\#1169322) as part of the Global Alliance for Chronic Disease Program on scaling up interventions to reduce hypertension and diabetes. CJ is supported by a National Heart Foundation Postdoctoral Fellowship (HF101945); JW is supported by a National Heart Foundation Career Development Fellowship (\#1082924), and through an NHMRC Centre of Research Excellence on food policy interventions to reduce salt (\#1117300).

Conflicts of Interest: The authors have no conflicts of interest to disclose.

Supplementary Table S1. Compliance of International and National manufacturers producing eligible products with voluntary Fijian sodium reformulation targets.

\begin{tabular}{|c|c|c|c|c|c|c|c|c|}
\hline \multirow[b]{2}{*}{ Category } & \multirow[b]{2}{*}{$\begin{array}{l}\text { Total } \\
\text { Produ } \\
\text { cts }(n)\end{array}$} & \multicolumn{7}{|c|}{ Nutrient Labelled $n(\%)$} \\
\hline & & Energy & Protein & $\begin{array}{l}\text { Carbohyd } \\
\text { rate }\end{array}$ & $\begin{array}{c}\text { Saturated } \\
\text { Fat }\end{array}$ & $\begin{array}{c}\text { Trans } \\
\text { Fat }\end{array}$ & $\begin{array}{c}\text { Mono- } \\
\text { Unsatura } \\
\text { ted Fat }\end{array}$ & $\begin{array}{c}\text { Poly- } \\
\text { Unsatura } \\
\text { ted Fat }\end{array}$ \\
\hline $\begin{array}{l}\text { Bread and bakery } \\
\text { products }\end{array}$ & 517 & $\begin{array}{c}506 \\
(97.9)\end{array}$ & $\begin{array}{c}506 \\
(97.9)\end{array}$ & $506(97.9)$ & $492(95.2)$ & $\begin{array}{c}283 \\
(54.7)\end{array}$ & $58(11.2)$ & $58(11.2)$ \\
\hline Bread & 54 & $\begin{array}{c}54 \\
(100.0)\end{array}$ & $\begin{array}{c}54 \\
(100.0)\end{array}$ & $54(100.0)$ & $50(92.6)$ & $\begin{array}{c}29 \\
(53.7)\end{array}$ & $0(0.0)$ & $0(0.0)$ \\
\hline Cakes, muffins and pastries & 121 & $\begin{array}{c}121 \\
(100.0)\end{array}$ & $\begin{array}{c}121 \\
(100.0)\end{array}$ & $121(100.0)$ & $\begin{array}{c}121 \\
(100.0)\end{array}$ & $\begin{array}{c}72 \\
(59.5)\end{array}$ & $11(9.1)$ & $11(9.1)$ \\
\hline Savoury biscuits & 104 & $\begin{array}{c}103 \\
(99.0)\end{array}$ & $\begin{array}{c}103 \\
(99.0)\end{array}$ & $103(99.0)$ & $102(98.1)$ & $\begin{array}{c}43 \\
(41.3)\end{array}$ & $22(21.2)$ & $22(21.2)$ \\
\hline Sweet biscuits & 238 & $\begin{array}{c}228 \\
(95.8)\end{array}$ & $\begin{array}{c}228 \\
(95.8)\end{array}$ & $228(95.8)$ & $219(92.0)$ & $\begin{array}{c}139 \\
(58.4)\end{array}$ & $25(10.5)$ & 25 (10.5) \\
\hline Cereal and grain products & 462 & $\begin{array}{c}454 \\
(98.3)\end{array}$ & $\begin{array}{c}453 \\
(98.1)\end{array}$ & 453 (98.1) & 423 (91.6) & $\begin{array}{c}205 \\
(44.4)\end{array}$ & $98(21.2)$ & $98(21.2)$ \\
\hline Breakfast cereals & 179 & $\begin{array}{l}178 \\
(99.4)\end{array}$ & $\begin{array}{c}178 \\
(99.4)\end{array}$ & $178(99.4)$ & $176(98.3)$ & $\begin{array}{c}81 \\
(45.3)\end{array}$ & $53(29.6)$ & $53(29.6)$ \\
\hline Cereal and nut-based bars & 53 & $\begin{array}{c}53 \\
(100.0)\end{array}$ & $\begin{array}{c}53 \\
(100.0)\end{array}$ & $53(100.0)$ & $53(100.0)$ & $\begin{array}{c}16 \\
(30.2)\end{array}$ & $12(22.6)$ & $12(22.6)$ \\
\hline Noodles & 59 & $\begin{array}{c}55 \\
(93.2)\end{array}$ & $\begin{array}{c}55 \\
(93.2)\end{array}$ & $55(93.2)$ & $51(86.4)$ & $\begin{array}{c}30 \\
(50.8)\end{array}$ & $9(15.3)$ & $9(15.3)$ \\
\hline $\begin{array}{l}\text { Other cereal and grain } \\
\text { products }\end{array}$ & 82 & $\begin{array}{c}79 \\
(96.3)\end{array}$ & $\begin{array}{c}78 \\
(95.1)\end{array}$ & $78(95.1)$ & $63(76.8)$ & $\begin{array}{c}35 \\
(42.7)\end{array}$ & $13(15.9)$ & $13(15.9)$ \\
\hline Pasta & 44 & $\begin{array}{c}44 \\
(100.0)\end{array}$ & $\begin{array}{c}44 \\
(100.0)\end{array}$ & $44(100.0)$ & $40(90.9)$ & $\begin{array}{c}12 \\
(27.3)\end{array}$ & $1(2.3)$ & $1(2.3)$ \\
\hline Rice & 45 & $\begin{array}{c}45 \\
(100.0)\end{array}$ & $\begin{array}{c}45 \\
(100.0)\end{array}$ & $45(100.0)$ & $40(88.9)$ & $\begin{array}{c}31 \\
(68.9)\end{array}$ & $10(22.2)$ & $10(22.2)$ \\
\hline Confectionery & 388 & $\begin{array}{l}378 \\
(97.4)\end{array}$ & $\begin{array}{l}379 \\
(97.7)\end{array}$ & $369(95.1)$ & $342(88.1)$ & $\begin{array}{c}85 \\
(21.9)\end{array}$ & $10(2.6)$ & $10(2.6)$ \\
\hline Chocolate and sweets & 364 & $\begin{array}{c}354 \\
(97.3)\end{array}$ & $\begin{array}{c}355 \\
(97.5)\end{array}$ & $345(94.8)$ & $324(89.0)$ & $\begin{array}{c}79 \\
(21.7)\end{array}$ & $7(1.9)$ & $7(1.9)$ \\
\hline Jelly & 24 & $\begin{array}{c}24 \\
(100.0)\end{array}$ & $\begin{array}{c}24 \\
(100.0)\end{array}$ & $24(100.0)$ & $18(75.0)$ & $6(25.0)$ & $3(12.5)$ & $3(12.5)$ \\
\hline Convenience foods & 60 & $\begin{array}{c}60 \\
(100.0)\end{array}$ & $\begin{array}{c}60 \\
(100.0)\end{array}$ & $60(100.0)$ & $59(98.3)$ & $\begin{array}{c}12 \\
(20.0)\end{array}$ & $4(6.7)$ & $4(6.7)$ \\
\hline
\end{tabular}




\begin{tabular}{|c|c|c|c|c|c|c|c|c|}
\hline Ready meals & 15 & $\begin{array}{c}15 \\
(100.0)\end{array}$ & $\begin{array}{c}15 \\
(100.0)\end{array}$ & $15(100.0)$ & $15(100.0)$ & $5(33.3)$ & $2(13.3)$ & $2(13.3)$ \\
\hline Soup & 45 & $\begin{array}{c}45 \\
(100.0)\end{array}$ & $\begin{array}{c}45 \\
(100.0)\end{array}$ & $45(100.0)$ & $44(97.8)$ & $7(15.6)$ & $2(4.4)$ & $2(4.4)$ \\
\hline Dairy & 348 & $\begin{array}{l}346 \\
(99.4)\end{array}$ & $\begin{array}{c}346 \\
(99.4)\end{array}$ & 344 (98.9) & $332(95.4)$ & $\begin{array}{c}143 \\
(41.1)\end{array}$ & $81(23.3)$ & $81(23.3)$ \\
\hline Cheese & 58 & $\begin{array}{c}58 \\
(100.0)\end{array}$ & $\begin{array}{c}58 \\
(100.0)\end{array}$ & $58(100.0)$ & $58(100.0)$ & $\begin{array}{c}22 \\
(37.9)\end{array}$ & $4(6.9)$ & $4(6.9)$ \\
\hline Cream & 26 & $\begin{array}{c}26 \\
(100.0)\end{array}$ & $\begin{array}{c}26 \\
(100.0)\end{array}$ & $26(100.0)$ & $26(100.0)$ & $\begin{array}{c}18 \\
(69.2)\end{array}$ & $2(7.7)$ & $2(7.7)$ \\
\hline Desserts & 11 & $\begin{array}{c}11 \\
(100.0)\end{array}$ & $\begin{array}{c}11 \\
(100.0)\end{array}$ & $11(100.0)$ & $11(100.0)$ & $4(36.4)$ & $1(9.1)$ & $1(9.1)$ \\
\hline Ice cream and edible ices & 112 & $\begin{array}{c}110 \\
(98.2)\end{array}$ & $\begin{array}{c}110 \\
(98.2)\end{array}$ & $109(97.3)$ & $100(89.3)$ & $\begin{array}{c}20 \\
(17.9)\end{array}$ & $17(15.2)$ & $17(15.2)$ \\
\hline Milk & 87 & $\begin{array}{c}87 \\
(100.0)\end{array}$ & $\begin{array}{c}87 \\
(100.0)\end{array}$ & $86(98.9)$ & $83(95.4)$ & $\begin{array}{c}57 \\
(65.5)\end{array}$ & $35(40.2)$ & $35(40.2)$ \\
\hline $\begin{array}{l}\text { Yoghurt and yoghurt } \\
\text { drinks }\end{array}$ & 54 & $\begin{array}{c}54 \\
(100.0)\end{array}$ & $\begin{array}{c}54 \\
(100.0)\end{array}$ & $54(100.0)$ & $54(100.0)$ & $\begin{array}{c}22 \\
(40.7)\end{array}$ & $22(40.7)$ & $22(40.7)$ \\
\hline $\begin{array}{l}\text { Edible oils and oil } \\
\text { emulsions }\end{array}$ & 105 & $\begin{array}{c}105 \\
(100.0)\end{array}$ & $\begin{array}{c}103 \\
(98.1)\end{array}$ & $104(99.0)$ & $91(86.7)$ & $\begin{array}{c}65 \\
(61.9)\end{array}$ & $79(75.2)$ & 74 (70.5) \\
\hline Fish and fish products & 116 & $\begin{array}{c}111 \\
(95.7)\end{array}$ & $\begin{array}{c}114 \\
(98.3)\end{array}$ & $111(95.7)$ & $112(96.6)$ & $\begin{array}{c}72 \\
(62.1)\end{array}$ & $46(39.7)$ & $46(39.7)$ \\
\hline Fruit and vegetables & 678 & $\begin{array}{c}666 \\
(98.2)\end{array}$ & $\begin{array}{c}669 \\
(98.7)\end{array}$ & $658(97.1)$ & $630(92.9)$ & $\begin{array}{c}212 \\
(31.3)\end{array}$ & $89(13.1)$ & $89(13.1)$ \\
\hline Fruit & 125 & $\begin{array}{c}125 \\
(100.0)\end{array}$ & $\begin{array}{c}125 \\
(100.0)\end{array}$ & $124(99.2)$ & $123(98.4)$ & $\begin{array}{c}28 \\
(22.4)\end{array}$ & $8(6.4)$ & $8(6.4)$ \\
\hline Jam and marmalades & 81 & $\begin{array}{c}78 \\
(96.3)\end{array}$ & $\begin{array}{c}81 \\
(100.0)\end{array}$ & $71(87.7)$ & $63(77.8)$ & $\begin{array}{c}19 \\
(23.5)\end{array}$ & $13(16.0)$ & $13(16.0)$ \\
\hline Nuts and seeds & 140 & $\begin{array}{c}138 \\
(98.6)\end{array}$ & $\begin{array}{c}138 \\
(98.6)\end{array}$ & $138(98.6)$ & $127(90.7)$ & $\begin{array}{c}60 \\
(42.9)\end{array}$ & $33(23.6)$ & $33(23.6)$ \\
\hline Vegetables & 332 & $\begin{array}{c}325 \\
(97.9)\end{array}$ & $\begin{array}{c}325 \\
(97.9)\end{array}$ & 325 (97.9) & 317 (95.5) & $\begin{array}{c}105 \\
(31.6)\end{array}$ & $35(10.5)$ & 35 (10.5) \\
\hline Meat and meat products & 105 & $\begin{array}{c}90 \\
(85.7)\end{array}$ & $\begin{array}{c}90 \\
(85.7)\end{array}$ & $81(77.1)$ & $81(77.1)$ & $\begin{array}{c}24 \\
(22.9)\end{array}$ & $5(4.8)$ & $5(4.8)$ \\
\hline Non-alcoholic beverages & 487 & $\begin{array}{c}472 \\
(96.9)\end{array}$ & $\begin{array}{c}471 \\
(96.7)\end{array}$ & $471(96.7)$ & $354(72.7)$ & $\begin{array}{c}134 \\
(27.5)\end{array}$ & $39(8.0)$ & $39(8.0)$ \\
\hline Beverage mixes & 38 & $\begin{array}{c}38 \\
(100.0)\end{array}$ & $\begin{array}{c}35 \\
(92.1)\end{array}$ & $38(100.0)$ & $20(52.6)$ & $\begin{array}{c}15 \\
(39.5)\end{array}$ & $0(0.0)$ & $0(0.0)$ \\
\hline Coffee and tea & 51 & $\begin{array}{c}51 \\
(100.0)\end{array}$ & $\begin{array}{c}51 \\
(100.0)\end{array}$ & $51(100.0)$ & $45(88.2)$ & $\begin{array}{c}27 \\
(52.9)\end{array}$ & $5(9.8)$ & $5(9.8)$ \\
\hline Cordials & 26 & $\begin{array}{c}26 \\
(100.0)\end{array}$ & $\begin{array}{c}26 \\
(100.0)\end{array}$ & $26(100.0)$ & $14(53.8)$ & $\begin{array}{c}10 \\
(38.5)\end{array}$ & $4(15.4)$ & $4(15.4)$ \\
\hline $\begin{array}{l}\text { Electrolyte and energy } \\
\text { drinks }\end{array}$ & 32 & $\begin{array}{c}27 \\
(84.4)\end{array}$ & $\begin{array}{c}27 \\
(84.4)\end{array}$ & $27(84.4)$ & $22(68.8)$ & $3(9.4)$ & $0(0.0)$ & $0(0.0)$ \\
\hline Fruit and vegetable juices & 228 & $\begin{array}{c}222 \\
(97.4)\end{array}$ & $\begin{array}{c}224 \\
(98.2)\end{array}$ & $221(96.9)$ & $178(78.1)$ & $\begin{array}{c}61 \\
(26.8)\end{array}$ & $14(6.1)$ & $14(6.1)$ \\
\hline Soft drinks & 94 & $\begin{array}{c}92 \\
(97.9)\end{array}$ & $\begin{array}{c}92 \\
(97.9)\end{array}$ & $92(97.9)$ & $70(74.5)$ & $\begin{array}{c}14 \\
(14.9)\end{array}$ & $12(12.8)$ & $12(12.8)$ \\
\hline Waters (flavoured) & 18 & $\begin{array}{c}16 \\
(88.9)\end{array}$ & $\begin{array}{c}16 \\
(88.9)\end{array}$ & $16(88.9)$ & $5(27.8)$ & $4(22.2)$ & $4(22.2)$ & $4(22.2)$ \\
\hline $\begin{array}{l}\text { Sauces, dressings, spreads } \\
\text { and dips }\end{array}$ & 628 & $\begin{array}{c}624 \\
(99.4)\end{array}$ & $\begin{array}{c}622 \\
(99.0)\end{array}$ & $615(97.9)$ & $528(84.1)$ & $\begin{array}{c}193 \\
(30.7)\end{array}$ & $42(6.7)$ & $42(6.7)$ \\
\hline $\begin{array}{l}\text { Mayonnaise and salad } \\
\text { dressings }\end{array}$ & 57 & $\begin{array}{c}57 \\
(100.0)\end{array}$ & $\begin{array}{c}55 \\
(96.5)\end{array}$ & $57(100.0)$ & $47(82.5)$ & $\begin{array}{c}16 \\
(28.1)\end{array}$ & $6(10.5)$ & $6(10.5)$ \\
\hline Sauces & 416 & $\begin{array}{c}414 \\
(99.5)\end{array}$ & $\begin{array}{c}414 \\
(99.5)\end{array}$ & 408 (98.1) & $333(80.0)$ & $\begin{array}{c}117 \\
(28.1)\end{array}$ & $15(3.6)$ & $15(3.6)$ \\
\hline Spreads and dips & 155 & $\begin{array}{l}153 \\
(98.7)\end{array}$ & $\begin{array}{l}153 \\
(98.7)\end{array}$ & $150(96.8)$ & 148 (95.5) & $\begin{array}{c}60 \\
(38.7)\end{array}$ & $21(13.5)$ & $21(13.5)$ \\
\hline Snackfoods & 290 & $\begin{array}{c}275 \\
(94.8)\end{array}$ & $\begin{array}{c}275 \\
(94.8)\end{array}$ & $274(94.5)$ & $250(86.2)$ & $\begin{array}{c}193 \\
(66.6)\end{array}$ & $118(40.7)$ & $118(40.7)$ \\
\hline Special foods & 26 & $\begin{array}{c}25 \\
(96.2)\end{array}$ & $\begin{array}{c}25 \\
(96.2)\end{array}$ & $25(96.2)$ & $4(15.4)$ & $3(11.5)$ & $0(0.0)$ & $0(0.0)$ \\
\hline $\begin{array}{l}\text { Sugars, honey and related } \\
\text { products }\end{array}$ & 68 & $\begin{array}{c}68 \\
(100.0)\end{array}$ & $\begin{array}{c}64 \\
(94.1)\end{array}$ & $68(100.0)$ & $52(76.5)$ & $\begin{array}{c}21 \\
(30.9)\end{array}$ & $15(22.1)$ & $15(22.1)$ \\
\hline $\begin{array}{l}\text { Dessert additions and } \\
\text { toppings }\end{array}$ & 42 & $\begin{array}{c}42 \\
(100.0)\end{array}$ & $\begin{array}{c}41 \\
(97.6)\end{array}$ & $42(100.0)$ & $34(81.0)$ & $\begin{array}{c}13 \\
(31.0)\end{array}$ & $9(21.4)$ & $9(21.4)$ \\
\hline
\end{tabular}




\begin{tabular}{lcccccccc} 
Honey and syrup & 26 & $\begin{array}{c}26 \\
(100.0)\end{array}$ & $\begin{array}{c}23 \\
(88.5)\end{array}$ & $26(100.0)$ & $18(69.2)$ & $8(30.8)$ & $6(23.1)$ & $6(23.1)$ \\
\hline \multirow{2}{*}{ Total } & \multirow{2}{*}{$\mathbf{4 2 7 8}$} & $\begin{array}{c}\mathbf{4 1 8 0} \\
\mathbf{( 9 7 . 7 )}\end{array}$ & $\begin{array}{c}\mathbf{4 1 7 7} \\
\mathbf{( 9 7 . 6 )}\end{array}$ & $\mathbf{4 1 3 9 ( \mathbf { 9 6 . 8 } )}$ & $\begin{array}{c}\mathbf{3 7 5 0} \\
\mathbf{( 8 7 . 7 )}\end{array}$ & $\begin{array}{c}\mathbf{1 6 4 5} \\
\mathbf{( 3 8 . 5 )}\end{array}$ & $\mathbf{6 8 4} \mathbf{( \mathbf { 1 6 . 0 ) }}$ & $\mathbf{6 7 9 ( \mathbf { 1 5 . 9 ) }}$ \\
\hline
\end{tabular}

\section{References}

1. World Health Organization. Western Pacific Regional Action Plan for the Prevention and Control of Noncommunicable Diseases (2014-2020); WHO Regional Office for the Western Pacific: Manila, Philippines, 2014.

2. World Health Organization. Non-communicable Disease (NCD) Country Profile. Available online: https://wwwwhoint/nmh/countries/fji_enpdf?ua=.2018 (23 September 2020).

3. Snowdon, W.; Thow, A.M. Trade policy and obesity prevention: Challenges and innovation in the Pacific Islands. Obes. Rev. Off. J. Int. Assoc. Study Obes. 2013, 14 (Suppl. 2), 150-158.

4. Ministry of Health and Medical Services National Food and Nutrition Centre. Fiji National Nutrition Survey 2015; 2018.

5. The George Instittue for Global Health. State of the Food Supply Fiji; University TPRCftPoOaNDaFN, ed.; 2019.

6. Charlton, K.E.; Russell, J.; Gorman, E.; Hanich, Q.; Delisle, A.; Campbell, B.; Bell, J. Fish, food security and health in Pacific Island countries and territories: A systematic literature review. BMC Public Health 2016, 16, 285.

7. Thow, A.M.; Heywood, P.; Schultz, J.; Quested, C.; Jan, S.; Colagiuri, S. Trade and the nutrition transition: Strengthening policy for health in the Pacific. Ecol. Food Nutr. 2011, 50, 18-42.

8. Reeve, E.; Thow, A.; Bell, C.; Soti-Ulberg, C. Identifying opportunities to strengthen school food environments in the Pacific: A case study in Samoa. BMC Public Health 2019, doi:10.21203/rs.2.16247/v2.

9. Webster, J.; Pillay, A.; Suku, A.; Gohil, P.; Santos, J.A.; Schultz, J.; Wate, J.; Trieu, K.; Hope, S.; Snowdon, W.; et al. Process Evaluation and Costing of a Multifaceted Population-Wide Intervention to Reduce Salt Consumption in Fiji. Nutrients 2018, 10, 155.

10. Thow, A.M.; Downs, S.; Jan, S. A systematic review of the effectiveness of food taxes and subsidies to improve diets: Understanding the recent evidence. Nutr. Rev. 2014, 72, 551-565.

11. World Health Organization. Global action plan for the prevention and control of noncommunicable diseases 20132020; World Health Organization: Geneva, Switzerland, 2013.

12. Downs, S.M.; Christoforou, A.; Snowdon, W.; Dunford, E.; Hoejskov, P.; Legetic, B.; Campbell, N.; Webster, J. Setting targets for salt levels in foods: A five-step approach for low-and middle-income countries. Food Policy 2015, 55, 101-108.

13. Fiji NFaNCMoHaMS. Health Minister Calls on the Food Industry to Reduce Salt Levels in Processed Foods in Fiji; Food Industry Salt Action Consultation: 2010.

14. Parliament of the Fiji Islands. Food Safety Act 2003; 2003.

15. Parliament of the Fiji Islands. Food Safety Regulations 2009; 2009.

16. Parliament of the Fiji Islands. Food and Safety (Amendment) Regulations; Services MoHaM, ed.; Government of Fiji: 2014.

17. Dunford, E.; Trevena, H.; Goodsell, C.; Ng, K.H.; Webster, J.; Millis, A.; Goldstein, S.; Hugueniot, O.; Neal, B. FoodSwitch: A Mobile Phone App to Enable Consumers to Make Healthier Food Choices and Crowdsourcing of National Food Composition Data. JMIR Mhealth Uhealth 2014, 2, e37.

18. Dunford, E.; Webster, J.; Metzler, A.B.; Czernichow, S.; Ni Mhurchu, C.; Wolmarans, P.; Snowdon, W.; L'Abbe, M.; Li, N.; Maulik, P.K.; et al. International collaborative project to compare and monitor the nutritional composition of processed foods. Eur. J. Prev. Cardiol. 2012, 19, 1326-1332.

Publisher's Note: MDPI stays neutral with regard to jurisdictional claims in published maps and institutional affiliations. 
(C) 2020 by the authors. Submitted for possible open access publication under the terms and conditions of the Creative Commons Attribution (CC BY) license (http://creativecommons.org/licenses/by/4.0/). 